\title{
Contributions of the nucleus accumbens and its subregions to different aspects of risk-based decision making
}

\author{
Colin M. Stopper $\cdot$ Stan B. Floresco
}

Published online: 15 December 2010

(C) Psychonomic Society, Inc. 2010

\begin{abstract}
The nucleus accumbens (NAc) has been implicated in mediating different forms of decision making in humans and animals. In the present study, we observed that inactivation of the rat NAc, via infusion of GABA agonists, reduced preference for a large/risky option and increased response latencies on a probabilistic discounting task. Discrete inactivations of the NAc shell and core revealed further differences between these regions in mediating choice and response latencies, respectively. The effect on choice was attributable to reduced win-stay performance (i.e., choosing risky after a being rewarded for a risky choice on a preceding trial). Moreover, NAc inactivation altered choice only when the large/risky option had greater long-term value, in terms of the amount of food that could be obtained over multiple trials relative to the small/certain option. Inactivation of the NAc or the shell subregion also slightly reduced preference for larger rewards on a reward magnitude discrimination. Thus, the NAc seems to play a small role in biasing choice toward larger rewards, but its contribution to behavior is amplified when delivery of these rewards is uncertain, helping to direct response selection toward more favorable outcomes.
\end{abstract}

Keywords Ventral striatum - Decision making .

Probabilistic discounting $\cdot$ Reversible inactivation $\cdot$ Rat

The capacity to make advantageous decisions is dependent upon the ability to evaluate the relative costs and benefits associated with different actions, in order to estimate which

C. M. Stopper $\cdot$ S. B. Floresco $(\bowtie)$

University of British Columbia,

2136 West Mall,

Vancouver, BC, Canada V6T 1Z4

e-mail: floresco@psych.ubc.ca option may yield outcomes of greater value. Costs associated with certain rewards may take multiple forms, including delaying the delivery of the reward, requiring more effort to obtain it, or making the reward probabilistic or "risky." Decisions under risk can involve choice between a smaller but more certain reward and a larger, probabilistic reward. In humans, real-world situations requiring assessments of the relative risks and rewards associated with different options include playing the stock market or gambling at a casino. Functional imaging studies employing laboratory tasks that share similarities with these types of decisions have revealed that the processes involved activate distributed cortical and subcortical networks. Of these brain regions, the nucleus accumbens (NAc) region of the ventral striatum has been heavily implicated in contributing to these types of judgments. For example, Kuhnen and Knutson (2005) conducted a financial risk task in which humans were asked to choose between two risky stocks, which yielded either a large gain or loss, or a safe bond that always yielded a small gain. Increased NAc activation preceded risky choices and risk-seeking mistakes. Similar results have been observed in numerous other studies employing various risk-based decision tasks, suggesting that NAc activation may bias choice toward riskier options associated with larger magnitudes of rewards (Knutson, Wimmer, Kuhnen, \& Winkielman, 2008; Matthews, Simmons, Lane, \& Paulus, 2004; Rao, Korczykowski, Pluta, Hoang, \& Detre, 2008; Samanez-Larkin, Kuhnen, Yoo, \& Knutson, 2010).

The notion that the NAc plays a critical role in cost/ benefit decision making is further supported by studies employing experimental animals. Many of these studies have focused on the contribution of this nucleus to effortor delay-related judgments. Thus, lesion or inactivation of the NAc core reduces the preference to work harder or wait longer for larger rewards (Cardinal, Pennicott, Sugathapala, Robbins, \& Everitt, 2001; Ghods-Sharifi \& Floresco 2010; 
Hauber \& Sommer, 2009; Pothuizen, Jongen-Rêlo, Feldon, $\&$ Yee, 2005). These forms of decision making are also altered by manipulations of the basolateral amygdala (Ghods-Sharifi, St. Onge, \& Floresco, 2009; Winstanley, Theobald, Cardinal, \& Robbins, 2004) or different regions of the prefrontal cortex (Mobini et al., 2002; Rudebeck, Walton, Smyth, Bannerman, \& Rushworth, 2006; Winstanley et al., 2004), regions that have also been implicated in decision-making processes in humans. However, in comparison, there have been somewhat fewer animal studies assessing the contribution of the NAc to risk/reward judgments. Cardinal and Howes (2005) utilized a probabilistic discounting task conducted in operant chambers. Rats chose between a small/certain lever that always delivered one pellet and a large/risky lever that delivered four pellets with a probability of $100 \%, 50 \%, 25 \%, 12.5 \%$, or $6.25 \%$, which decreased in a systematic manner across trial blocks. Normal animals adjusted their bias over a session accordingly, selecting the large/risky lever more often in early trial blocks and the small/certain lever during later trial blocks. Permanent, excitotoxic lesions to the NAc following training initially caused rats to be relatively indifferent to the two alternatives, choosing the large/risky option on $\sim 50 \%$ of trials across all probability blocks. With extended training, lesioned animals eventually biased their choice toward the small/certain lever primarily during the earlier trial blocks, when the large/risky option was more advantageous. However, a control experiment revealed that lesions of the NAc did not significantly reduce choice of a larger reinforcer when the probability of receiving it was fixed at $100 \%$. These findings led to the conclusion that the NAc is involved specifically in the processing of differently valued rewards under conditions of uncertainty and that lesions of this nucleus induce risk aversion.

It is now well-established that the NAc can be subdivided into core and shell subregions, based on a variety of neurochemical and anatomical characteristics (Brog, Salyapongse, Deutch, \& Zahm, 1993; Floresco, 2007; Groenewegen et al., 1991; Ikemoto \& Panksepp, 1999; Mogenson, Brudzynski, Wu, Yang, \& Yim, 1993; Pennartz, Groenewegen, \& Lopes Da Silva, 1994). Accordingly, lesions of the NAc core or shell have been reported to produce dissociable effects on a variety of behaviors, including instrumental action, latent inhibition, set shifting, and cue-induced reinstatement of food-seeking behavior (Corbit, Muir, \& Balleine, 2001; Floresco, GhodsSharifi, Vexelman, \& Magyar, 2006; Floresco, McLaughlin, \& Haluk, 2008; Weiner, 2003). Studies of cost/benefit decision making using subregion-selective manipulations of the NAc have also identified dissociations between the core and the shell, with the core appearing to play a more critical role than the shell in mediating both delay- and effort-based decision making (Cardinal et al., 2001; Ghods-Sharifi \&
Floresco, 2010; Hauber \& Sommer, 2009; Pothuizen et al., 2005). With respect to risk-based decision making, Cardinal and Howes (2005) lesioned the more lateral portions of the NAc core, but these lesions also included considerable damage to the more medial portions of the shell. Thus, it is unclear whether the reduced preference for larger, probabilistic rewards induced by lesions of the NAc in this study was attributable primarily to cell loss in the NAc core or shell (or both).

The present study was conducted to further clarify the contribution that the NAc makes to risk-based decision making. We utilized a probabilistic discounting task similar to that we have used previously (Cardinal \& Howes, 2005) to investigate the roles of different regions of the prefrontal cortex, of the basolateral amygdala, and of dopamine in this form of decision making (GhodsSharifi et al., 2009; St. Onge \& Floresco, 2009, 2010). Since the effects of permanent lesions of the NAc on probabilistic discounting can vary over extended training, we used reversible inactivation of this nucleus in order to ascertain how a transient suppression of neural activity alters decision making in well-trained animals. An initial experiment employed relatively large inactivation of the entire NAc, and subsequent experiments used more discrete inactivations to determine which subregion (core or shell) played a greater role in mediating these effects. In these experiments, we also analyzed whether changes in choice behavior were due to a reduced tendency to select the risky option after obtaining a larger reward on previous trials (win-stay) or to an increased tendency to choose the small/ certain option after reward omission (lose-shift). Subsequent experiments further probed how NAc inactivation affected choice when reward probabilities remained constant over a session, and also how these treatments affected choice between larger versus smaller rewards.

\section{Method}

Animals

For these experiments, we used male Long Evans rats (Charles River Laboratories, Montreal, Canada) weighing 250-300 g at the beginning of training. On arrival, the rats were given 1 week to acclimatize to the colony and were then food restricted to $85 \%-90 \%$ of their free-feeding weight for 1 week before behavioral training and given ad libitum access to water for the duration of the experiment. Feeding occurred in the rats' home cages at the end of the experimental day, and body weights were monitored daily. All testing was in accordance with the Canadian Council on Animal Care and the Animal Care Committee of the University of British Columbia. 


\section{Apparatus}

Behavioral testing was conducted in 12 operant chambers $(30.5 \times 24 \times 21 \mathrm{~cm}$; MED Associates, St Albans, VT) enclosed in sound-attenuating boxes. The boxes were equipped with a fan that provided ventilation and masked extraneous noise. Each chamber was fitted with two retractable levers, one located on each side of a central food receptacle where food reinforcement $(45 \mathrm{mg}$; Bioserv, Frenchtown, NJ) was delivered by a pellet dispenser. The chambers were illuminated by a single $100-\mathrm{mA}$ house light located in the top center of the wall opposite the levers. Four infrared photobeams were mounted on the side of each chamber, and another photobeam was located in the food receptacle. Locomotor activity was indexed by the number of photobeam breaks that occurred during a session. All experimental data were recorded by personal computers connected to the chambers through an interface.

\section{Leverpress training}

Our initial training protocols were identical to those of St. Onge and Floresco (2009), as adapted from Cardinal, Robbins, and Everitt (2000). On the day before their first exposure to the operant chamber, rats were given approximately 25 food reward pellets in their home cage. On the first day of training, 2-3 pellets were delivered into the food cup and crushed pellets were placed on a lever before the animal was placed in the chamber. Rats were first trained under a fixed-ratio-1 schedule to a criterion of 60 pellets in $30 \mathrm{~min}$, first for one lever, and then repeated for the other lever (counterbalanced left/right between subjects). They were then trained on a simplified version of the full task. These 90 trial sessions began with the levers retracted and the operant chamber in darkness. Every $40 \mathrm{~s}$, a trial was initiated with the illumination of the house light and the insertion of one of the two levers into the chamber. If the rat failed to respond on the lever within $10 \mathrm{~s}$, the lever retracted and a single pellet was delivered with $50 \%$ probability. This procedure was used to familiarize the rats with the probabilistic nature of the full task. In every pair of trials, the left or right lever was presented once, and the order within the pair of trials was random. Rats were trained for approximately $3-5$ days to a criterion of 80 or more successful trials (i.e., $<10$ omissions).

\section{Decision-making tasks}

Risk-discounting task The primary task used in these studies has been described previously (Floresco \& Whelan, 2009; Ghods-Sharifi et al., 2009; St. Onge, Chiu, \& Floresco, 2010; St. Onge \& Floresco, 2009, 2010), which was originally modified from that described by Cardinal and Howes (2005) (see Fig. 1). Rats received daily sessions consisting of 72 trials, separated into four blocks of 18 trials. The entire session took $48 \mathrm{~min}$ to complete, and the animals were trained 5-7 days per week. A session began in darkness with both levers retracted (the intertrial state). A trial began every $40 \mathrm{~s}$ with the illumination of the house light and the insertion of one or both levers into the chamber. One lever was designated the large/risky lever, the other the small/certain lever, which remained consistent throughout training (counterbalanced left/right). If the rat did not respond within $10 \mathrm{~s}$ of lever presentation, the

a

Small/Certain Lever
(delivers 1 pellet

(delivers 1 pellet
on every press)
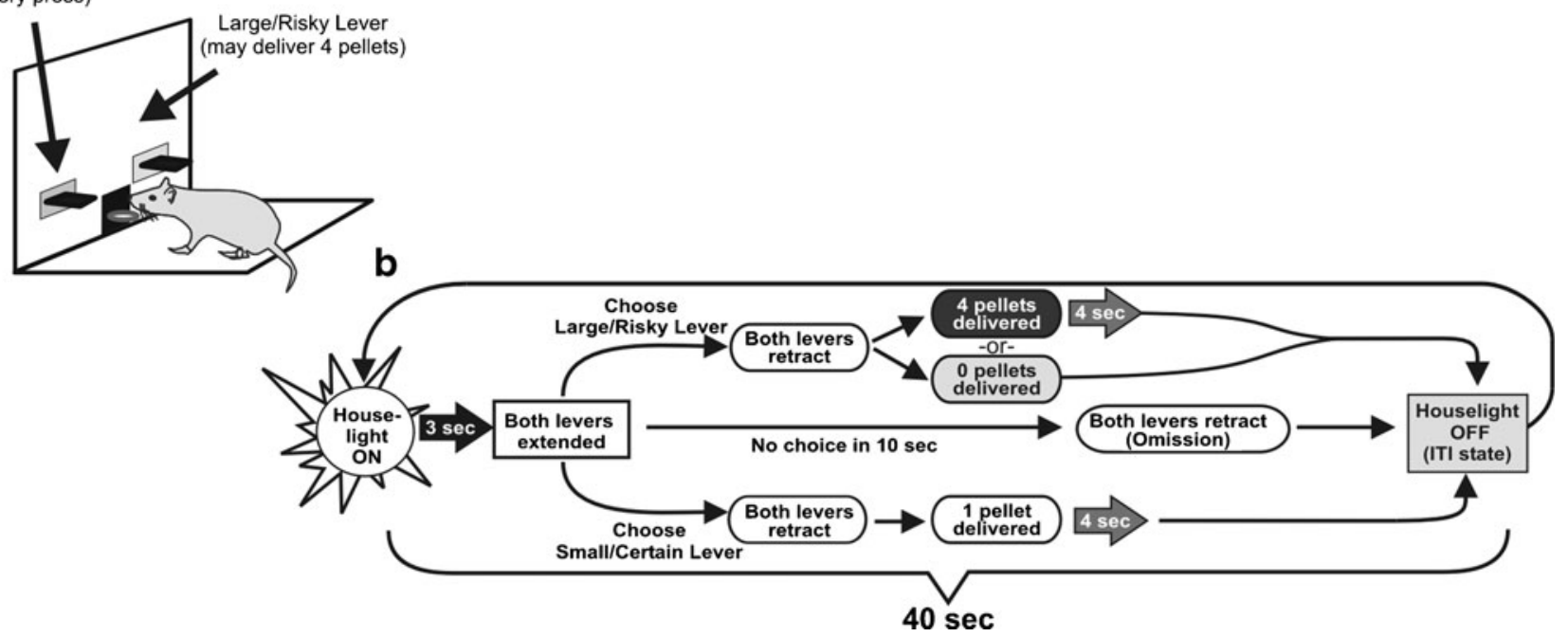

Fig. 1 Risk-discounting task design. (a) Cost/benefit contingencies associated with responding on either lever and (b) format of a single freechoice trial 
chamber was reset to the intertrial state until the next trial (omission). When a lever was chosen, both levers retracted. Choice of the small/certain lever always delivered one pellet with $100 \%$ probability; choice of the large/risky lever delivered four pellets, but with a particular probability that varied by blocks (see below). After a response was made and food delivered, the house light remained on for another $4 \mathrm{~s}$, after which the chamber reverted back to the intertrial state until the next trial. Multiple pellets were delivered $0.5 \mathrm{~s}$ apart. The four blocks consisted of 8 forced-choice trials where only one lever was presented (4 trials for each lever, randomized in pairs) permitting animals to learn the amount of food associated with each leverpress and the respective probability of receiving reinforcement over each block. This was followed by 10 free-choice trials, where both levers were presented and the animal chose between the small/certain or the large/risky lever. The probability of obtaining four pellets after pressing the large/risky lever was varied systematically across the four blocks: It was initially $100 \%$, and then $50 \%, 25 \%$, and $12.5 \%$, in that order. Thus, when the probability of obtaining the fourpellet reward was $100 \%$ or $50 \%$, this option would be more advantageous. At $25 \%$, it was arbitrary which lever the animal should choose, and at $12.5 \%$, the small/certain lever would be the more advantageous option in the long term.

Rats were trained on the task until, as a group, they (1) chose the large/risky lever during the first trial block (100\% probability) on at least $80 \%$ of successive trials, (2)chose the large/risky lever during the final trial block $(12.5 \%$ probability) on at most $60 \%$ of successive trials, and (3) demonstrated stable baseline levels of choice. Infusions were administered after a group of rats displayed stable patterns of choice for three consecutive days, assessed using a procedure similar to that described by Winstanley, Theobald, Dalley, and Robbins (2005) and Floresco, Tse, and Ghods-Sharifi (2008). In brief, the data from three consecutive sessions were analyzed with a repeated measures ANOVA with two within-subjects factors (Day and Trial Block). If the effect of block was significant at the $p<.05$ level but there was no main effect of day or Day $\mathrm{x}$ Block interaction (at the $p>.1$ level), animals were judged to have displayed stable baseline levels of discounting.

Risk discounting with fixed probabilities In a subsequent experiment, we employed a fixed-probability risk task we had used previously to investigate the contribution of the medial prefrontal cortex to this form of decision making (St. Onge \& Floresco, 2010). Rats were initially trained to press retractable levers for the risk-discounting task and then trained on a choice task. Each daily session started with 20 forced-choice trials, followed by 20 free-choice trials. As with the risk-discounting task, the small/certain lever always delivered one pellet with $100 \%$ probability. However, the probability of obtaining the larger, four-pellet reward after selection of the large/risky lever remained constant over the entire session. For the first phase of this experiment, this probability was set at $40 \%$. Rats were trained until they displayed stable levels of choice, after which they received their first round of counterbalanced microinfusions of saline and baclofen/muscimol. They were then retrained on the task with the probability of the large/ risky reward set to $10 \%$ for 15 days, after which they received a second round of counterbalanced microinfusions.

Reward magnitude discrimination task As we have done previously (Ghods-Sharifi et al., 2009), we determined a priori that if inactivation of the NAc or one of its subregions decreased preference for the large/risky lever on the risk-discounting task, separate groups of animals would be trained and tested on a reward magnitude discrimination task to determine whether this effect was due to an impairment in discriminating between reward magnitudes associated with the two levers. In these experiments, rats were trained to press retractable levers as in the riskdiscounting task, after which they were trained on the reward magnitude discrimination task. Here, rats chose between one lever that delivered one pellet and another that delivered four pellets. Both the small and large rewards were delivered immediately after a single response with $100 \%$ probability. A session consisted of four blocks of trials, with each block consisting of 2 forced-choice followed by 10 free-choice trials. After $\sim 15$ days of training, rats displayed a strong preference for the fourpellet option. They were implanted with guide cannulae and recovered for at least 7 days. After $\sim 5$ days of retraining, their choice behavior stabilized, and they received counterbalanced infusions on separate test days.

\section{Surgery and microinfusion protocol}

Rats were trained on their respective tasks until they displayed stable levels of choice, after which they were provided with food ad libitum for 1-3 days and were then subjected to surgery. Rats were anaesthetized with $100 \mathrm{mg} /$ $\mathrm{kg}$ ketamine hydrochloride and $7 \mathrm{mg} / \mathrm{kg}$ xylazine and implanted with bilateral 23-gauge stainless steel guide cannulae aimed at one of three coordinates using standard stereotaxic techniques. Some rats received implants aimed at the central portion of the NAc along the core/shell border, to inactivate both subregions (flat skull: anteroposterior $=$ $+1.5 \mathrm{~mm}$; mediolateral $= \pm 1.4 \mathrm{~mm}$; dorsoventral $=-5.9 \mathrm{~mm}$ from dura). For studies employing subregion-specific microinfusions, guide cannuale were aimed at either the NAc 
core (flat skull: anteroposterior $=+1.5 \mathrm{~mm}$; mediolateral $=$ $\pm 1.8 \mathrm{~mm}$; dorsoventral $=-5.9 \mathrm{~mm}$ from dura) or shell (flat skull: anteroposterior $=+1.6 \mathrm{~mm}$; mediolateral $= \pm 1.0 \mathrm{~mm}$; dorsoventral $=-5.9 \mathrm{~mm}$ from dura). Guide cannulae were held in place with stainless steel screws and dental acrylic. Thirty-gauge obdurators flush with the end of guide cannulae remained in place until the infusions were made. Rats were given at least 7 days to recover from surgery before testing. During this period, they were handled at least $5 \mathrm{~min}$ each day and were food restricted to $85 \%$ of their free-feeding weight.

Rats were subsequently trained on their respective task for at least 5 days until the group displayed stable levels of choice behavior for three consecutive days. One to two days before their first microinfusion test day, obdurators were removed, and a mock infusion procedure was conducted: Stainless steel injectors were placed in the guide cannulae for $2 \mathrm{~min}$, but no infusion was administered. The day after displaying stable discounting, the group received its first microinfusion test day.

A within-subjects design was used for all experiments. Inactivation was achieved by microinfusion of a solution containing the $\mathrm{GABA}_{\mathrm{B}}$ agonist baclofen and the $\mathrm{GABA}_{\mathrm{A}}$ agonist muscimol (Sigma Aldrich). Both drugs were dissolved in physiological saline, mixed separately at a concentration of $500 \mathrm{ng} / \mu \mathrm{l}$, and then combined into equal volumes so that the final concentration of each compound in solution was $250 \mathrm{ng} / \mu \mathrm{l}$. For inactivation of the entire NAc, drugs or saline were infused at a volume of $0.5 \mu \mathrm{l}$, so the final dose of baclofen and muscimol was $125 \mathrm{ng} / \mathrm{side}$. For subregion-specific inactivation of the shell or core, drugs or saline were infused at a volume of $0.3 \mu \mathrm{l}$, so the final dose of baclofen and muscimol was $75 \mathrm{ng} / \mathrm{side}$. Infusions of GABA agonists or saline were administered bilaterally via 30 -gauge injection cannulae that protruded $0.8 \mathrm{~mm}$ past the end of the guide cannulae, at a rate of $0.4 \mu \mathrm{l} / \mathrm{min}$ by a microsyringe pump. Injection cannulae were left in place for an additional $1 \mathrm{~min}$ to allow for diffusion. Each rat remained in its home cage for an additional 10-min period before behavioral testing. Previous studies using similar infusions of $0.3 \mu \mathrm{l}$ of baclofen/ muscimol solutions with similar concentrations have observed dissociable effects on behavior when GABA agonists have been infused into adjacent brain regions separated by $\sim 1 \mathrm{~mm}$ (Floresco et al., 2006; Floresco et al., 2008; Marquis, Killcross, \& Haddon, 2007; Moreira, Masson, Carvalho, \& Brandão, 2007), suggesting that functional spread of these treatments is likely $\leq 1 \mathrm{~mm}$ in diameter. Furthermore, neurophysiological studies have shown that administration of muscimol into the brain induces a near complete suppression of neural activity that lasts for over $2 \mathrm{~h}$ (van Duuren et al., 2007). Thus, infusion of a combination of both muscimol and baclofen would be expected to induce an inactivation that would persist over the duration of the test sessions used in the present study (25-48 $\mathrm{min})$.

On the first infusion test day, half of the rats in each group received saline infusions, and the other half received baclofen/muscimol. The next day, they received a baseline training day (no infusion). If, for any individual rat, choice of the large/risky lever deviated by $>15 \%$ from its preinfusion baseline, it received an additional day of training before the second infusion test. On the following day, rats received a second counterbalanced infusion of either saline or baclofen/muscimol.

\section{Histology}

After completion of behavioral testing, rats were euthanized in a carbon dioxide chamber. Brains were removed and fixed in a $4 \%$ formalin solution. The brains were frozen and sliced in 50- $\mu \mathrm{m}$ sections before being mounted and stained with Cresyl Violet. Placements were verified with reference to the neuroanatomical atlas of Paxinos and Watson, (1998). Data from rats whose placements were outside the borders of the NAc core or shell or encroached into the lateral ventricle were removed from the analysis. In general, animals with inaccurate placements did not display prominent changes in choice behavior following inactivation treatments relative to saline infusions. Core and shell placements were confined to their respective regions, with no evidence of diffusion into the other. Placements in the more central portion of the NAc were located along the border of the core and shell, which would be expected to diffuse across portions of both subregions (Fig. 2).

\section{Data analysis}

The primary dependent measure of interest was the proportion of choices directed toward the large/risky lever for each block of free-choice trials, factoring in trial omissions. For each block, this was calculated by dividing the number of choices of the large/risky lever by the total number of successful trials. Choice data were analyzed using two-way within-subjects ANOVAs, with Treatment and Trial Block as the within-subjects factors. In each of these analyses, the effect of trial block was always significant $(p<.001)$ on the risk-discounting task and will not be reported further. Response latencies on free-choice trials (i.e., the time elapsed between the insertion of the levers into the chambers and a subsequent leverpress) were analyzed with an ANOVA model similar to that used for choice data. Locomotor activity (i.e., photobeam breaks) and the number of trial omissions were analyzed with oneway repeated measures ANOVAs. 

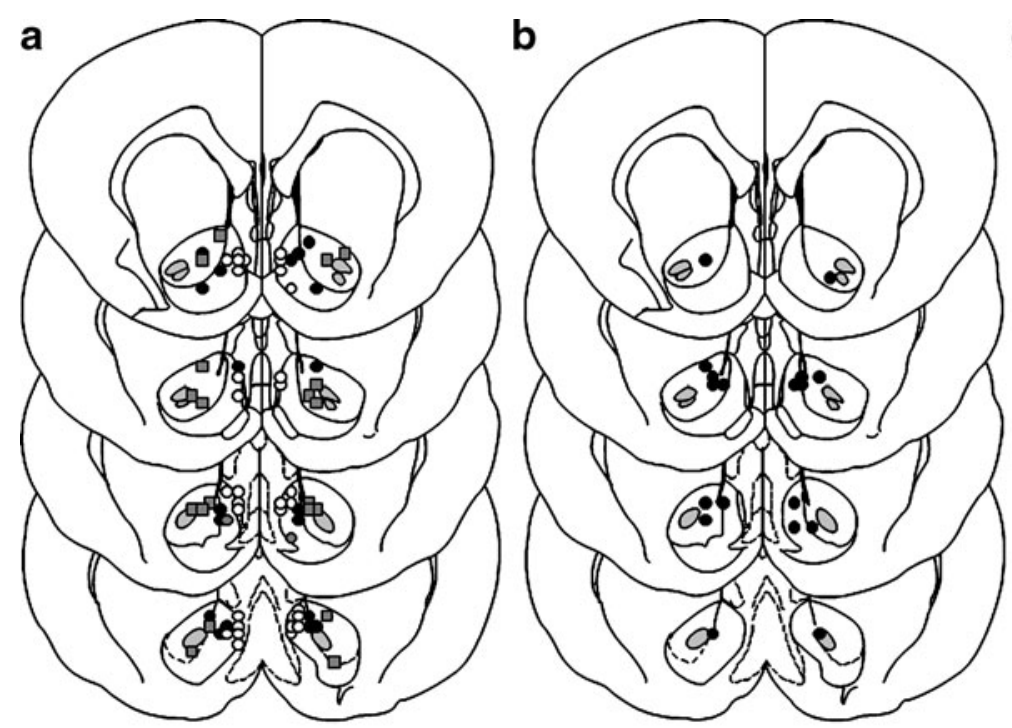

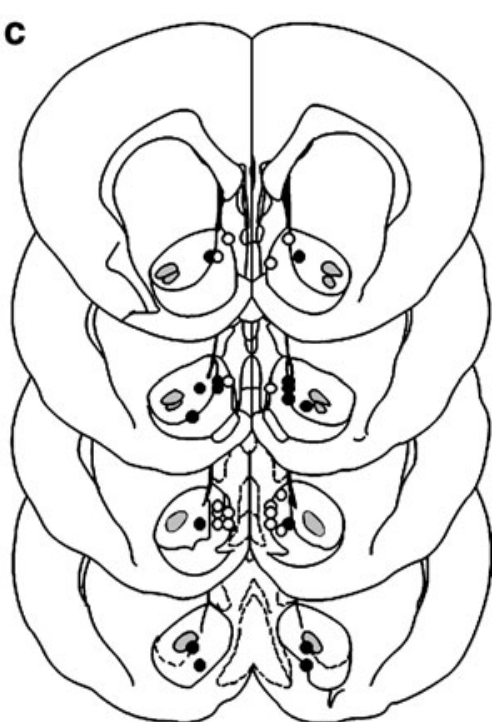

Fig. 2 Cannulae/injector placements for (a) the risk-discounting task, (b) the risk discounting with fixed probabilities task, and (c) the reward magnitude discrimination task. Black circles denote entire-
NAc placements, white circles denote shell placements, and gray squares denote core placements

\section{Results}

Experiment 1: Effects of inactivation of the NAc and its subregions on risk discounting

$N A c$ inactivations In our first experiment, we infused a larger dose and volume of baclofen/muscimol aimed at the border between the core and shell, in order to induce a broader inactivation of both subregions of this nucleus. Rats in this experiment were trained on the risk-discounting task for an average of 26 days prior to being implanted with guide cannulae into the central portion of the NAc, being retrained on the task, and receiving counterbalanced microinfusions. All of the rats in this experiment required only 1 day of retraining following the first infusion before receiving their second infusion treatment. Analysis of choice behavior following bilateral infusions of baclofen/ muscimol or saline into the NAc $(n=10)$ revealed a significant main effect of treatment $[F(1,9)=12.87$, $p<.01$; Fig. 3a] but no significant Treatment $\mathrm{x}$ Block interaction $[F(3,27)=1.76$, n.s. $]$. Relative to saline infusions, NAc inactivation caused a significant decrease in the proportion of choices directed toward the large/risky lever. Inspection of Fig. 3a reveals that this effect was apparent during the first, 100\%-probability block and persisted through the next two blocks. Despite the lack of a significant Treatment $\mathrm{x}$ Block interaction, this observed difference in the first three blocks prompted us to conduct an exploratory analysis. A paired-sample $t$ test comparing an average of the choice behavior over the first three blocks with behavior on the final block was conducted between 
a

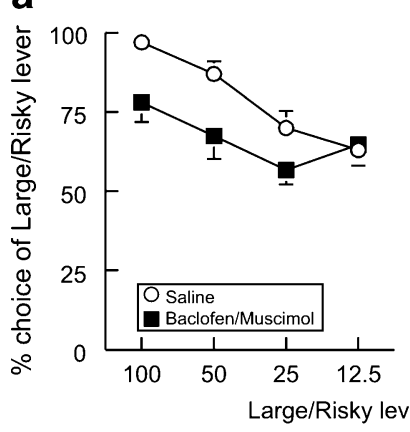

Fig. 3 The effects of inactivation of the NAc on risk discounting. (a) Choice data, expressed as proportions of choices on the large/risky lever during free-choice trials in each of the four probability blocks. Inactivation of the NAc reduced preference for the large/risky lever, most prominently in the first three trial blocks. (b) Inactivation of the NAc also increased response latencies, with this effect being statistically significant in the second and last blocks. (c) Win-stay/ lose-shift data. Win-stay data are displayed as the proportions of b

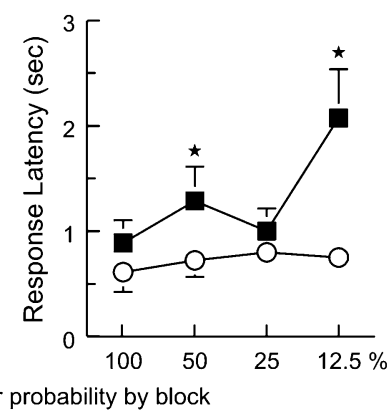

C

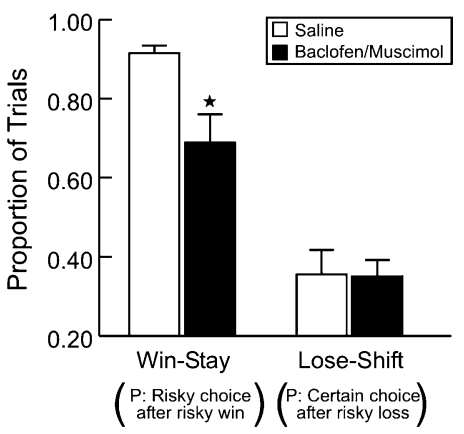

choices of the large/risky lever after selecting this lever on the preceding trial and obtaining the large reward (win). Lose-shift data are displayed as the proportions of choices of the small/certain lever following selection of the large/risky lever on the preceding trial and not obtaining reward (loss). Inactivation of the NAc selectively reduced win-stay tendencies. Stars denote a significant difference during a particular trial block (b) or a significant main effect of treatment at $p<.05$ (c) saline and inactivation. These analyses revealed a significant effect of treatment on the first three blocks $[t(9)=3.324, p<.01]$ but no effect on the final block $[t(9)=0.254$, n.s.]. Inactivation significantly increased response latencies $[F(1,9)=8.45, p<.05]$, primarily in the second and last blocks, as indicated by a significant Treatment $\mathrm{x}$ Block interaction $[F(3,27)=4.67, p<.01$; Fig. 3b].

We further analyzed response latencies as a function of choice type (large/risky vs. small/certain). The analysis revealed no Treatment $x$ Choice Type interaction $[F(1,9)=$ 1.01 , n.s.], indicating that NAc inactivation did not differentially affect response latencies when animals selected the risky versus the certain option. Note that other studies of cost/ benefit decision making associated with effort-related judgments have observed differences in response latencies when animals were presented with high- or low-effort options during forced-choice trials (Walton, Kennerley, Bannerman, Phillips, \& Rushworth, 2006). The discrepancy between that study and the present one may be related to either differences in free- versus forced-choice situations or differences in processing times when rats evaluate risk- versus effort-related costs.

Locomotor activity was significantly decreased following infusion of baclofen/muscimol relative to saline $[F(1,9)=23.71, p<.005$; Table 1]. Omissions were significantly increased following infusion of baclofen/ muscimol $[F(1,9)=7.98, p<.05$; Table 1$]$.

We further analyzed the proportions of "win-stay" and "lose-shift" trials to determine whether the effects of NAc inactivation on choice could be attributed to altered reward or negative-feedback sensitivity, respectively. Analysis of win-stay trials revealed a significant effect of treatment $[F(1,9)=9.77, p<.05$; Fig. 3c]. Specifically, under control conditions, rats displayed a strong tendency to select the risky lever after selecting this lever on the preceding trial and receiving a reward. However, inactivation of the NAc decreased the probability of choosing the risky option following a "win" on the large/risky lever. In contrast, lose-shift performance was not altered following inactivation of the NAc $[F(1,9)=0.00$, n.s $]$. Collectively, these results indicate that inactivation of the NAc reduces preference for the larger, uncertain rewards most prominently when it would be more advantageous to choose them over smaller, certain rewards. Furthermore, this effect appears to be due primarily to a decreased tendency to select the risky option following receipt of the large reward on the previous trial, as opposed to an exaggerated tendency to shift to the small/certain option after reward omission.

Table 1 Locomotion (number of photobeam breaks) and trial omission data obtained from Experiment 1 following control or inactivation treatments in the NAc and more discrete inactivation of shell and core subregions

\begin{tabular}{|c|c|c|c|c|}
\hline & \multicolumn{2}{|c|}{ Vehicle } & \multicolumn{2}{|c|}{ Inactivation } \\
\hline & $M$ & $S E$ & $M$ & $S E$ \\
\hline NAc: Locomotion & 1,976 & 135 & $1,375^{*}$ & 163 \\
\hline Trial omissions & 0.4 & 0.3 & $5.3^{*}$ & 0.02 \\
\hline NAc shell: Locomotion & 1,476 & 162 & 1,713 & 170 \\
\hline Trial omissions & 1.9 & 1.1 & 3.5 & 0.6 \\
\hline NAc core: Locomotion & 1,878 & 215 & $1,182 *$ & 187 \\
\hline Trial omissions & 0.6 & 0.3 & 6.3 & 3.1 \\
\hline
\end{tabular}

$* p<.05$ between vehicle and inactivation 
NAc shell inactivations Sixteen rats with accurate placement within the NAc shell were used in the analysis. These rats were trained for 24 days on the risk-discounting task, at which point they showed stable discounting behavior. They were subsequently implanted with cannulae aimed at the NAc shell, followed by retraining and counterbalanced infusions. Eight of the rats, 6 receiving saline as their first infusion and 2 receiving baclofen/muscimol as their first infusion, required a second baseline day between infusions for choice behavior to restabilize. Analysis of their choice behavior revealed a significant main effect of treatment $[F(1,15)=4.771, p<.05$; Fig. $4 \mathrm{a}]$ but no Treatment $\mathrm{x}$ Block interaction $[F(3,45)=0.978$, n.s.]. As was observed following larger infusions of baclofen/muscimol into the central NAc, inactivation of the NAc shell also decreased preference for the large/risky lever, although this effect was somewhat smaller than we had observed in the entire-NAc inactivation group. In contrast, analysis of the latency data did not yield either a significant main effect of treatment $[F(1,15)=0.35$, n.s. $]$ or a Treatment $\mathrm{x}$ Block interaction $[F(3,45)=0.353$, n.s.; Fig. $4 b]$. There was no significant

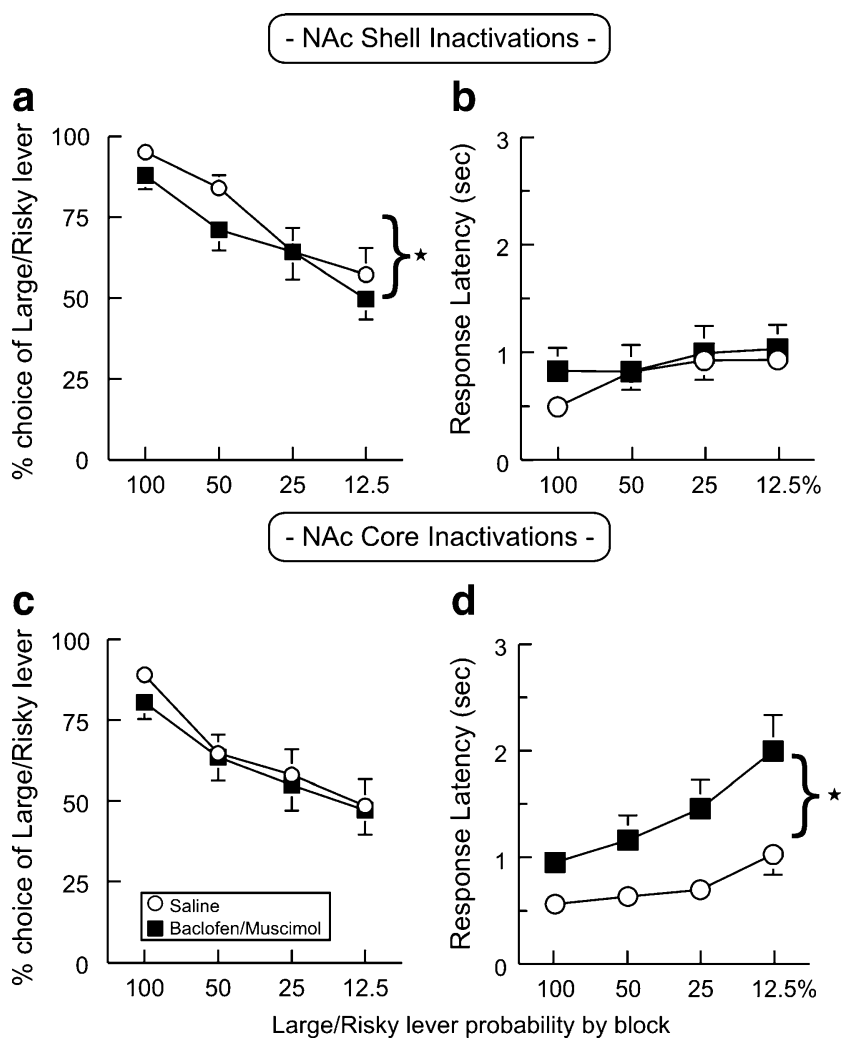

Fig. 4 Dissociable effects of selective inactivation of the NAc shell and core on risk discounting. Inactivation of the NAc shell reduced choice of the large/risky option (a), but did not affect response latencies (b). In contrast, inactivation of the NAc core did not affect choice (c) but did increase response latencies (d). Stars denote significant main effects of treatment at $p<.05$ effect on either locomotor activity $[F(1,15)=0.94$, n.s.; Table 1] or trial omissions $[F(1,15)=0.374$, n.s; Table 1].

Win-stay tendencies after inactivation of the NAc shell were similar to those observed after infusions of a larger volume of baclofen/muscimol into the entire NAc, such that inactivation of the NAc shell decreased the probability of choosing the risky option following a win on the large/risky lever $(M=.77 \pm .05)$ relative to saline treatment $(M=.87 \pm .04)$. Analysis of these data showed that this effect approached significance $[F(1,15)=4.01, p=.06]$. Again, lose-shift performance was not altered following inactivation of the NAc shell (saline, $M=.28 \pm .06$; inactivation, $M=.36 \pm .08$; $F(1,15)=1.11, \mathrm{n.s}]$. Thus, taken together, these findings imply that the reduced preference for the large/risky option induced by larger inactivation of the NAc were attributable primarily to suppression of neural activity within the NAc shell.

NAc core inactivations Data from 10 rats with accurate placements within the NAc core were included in the analysis. In stark contrast to what we had observed following inactivation of the NAc or more specific inactivation of the NAc shell, infusions of baclofen/ muscimol into the NAc core did not induce a significant change in choice behavior relative to saline treatment [main effect of treatment, $F(1,9)=0.28$, n.s.; Treatment $\mathrm{x}$ Block interaction, $F(3,27)=0.34$, n.s.; Fig. 4c]. Since these treatments did not significantly affect overall choice behavior, we did not conduct an analysis on win-stay/ lose-shift performance. However, as was observed following larger inactivations of the NAc (but not of the NAc shell), these treatments did significantly increase response latencies $[F(1,9)=12.56, p<.01$; Fig. $4 d]$, although in this analysis, we did not observe a significant Treatment $\mathrm{x}$ Block interaction $[F(3,27)=1.38$, n.s.]. Core inactivation also decreased locomotion $[F(1,9)=13.40, p<.01$; Table 1] but did not induce a statistically reliable increase in trial omissions $[F(1,9)=3.11$, n.s.; Table 1]. Taken together, these data suggest that the increased response latencies and decreased locomotion induced by larger inactivation of the NAc were attributable primarily to suppression of neural activity within the NAc core, whereas the reduced preference for the large/risky option was attributable primarily to suppression of neural activity within the NAc shell.

Experiment 2: Effects of inactivation of the NAc on decision making with fixed probabilities

Experiment 1 revealed that inactivation of the NAc reduced preference for the large/risky option, with this effect being most prominent when this option was more advantageous 
relative to the small/certain option. Experiment 2 employed a simplified version of the task in which the odds of obtaining the large reward remained constant over a session, in order to determine whether inactivation of the NAc induced a general decrease in preference for large/ risky rewards or a more selective effect under conditions in which the risky option was more advantageous.

Rats were trained on a simplified risk task where the probability of reinforcement on the large/risky lever was fixed at $40 \%$ over the entire session. Using these probabilities, selection of the large/risky lever would have a greater longterm value, yielding more reward over 20 free-choice trials (four pellets at $40 \%$ ) relative to the small/certain lever (one pellet at $100 \%$ ). Accordingly, after 12 days of training, rats displayed stable bias toward the large/risky lever, selecting this option on $\sim 75 \%$ of free-choice trials. They subsequently received their first round of counterbalanced infusions of saline and baclofen/musimol (125 ng each in $0.5 \mu \mathrm{l})$. Two rats receiving baclofen/muscimol for their first infusion required a second baseline training day for choice behavior to restabilize before their saline infusion. Analysis of their choice behavior revealed a significant main effect of treatment $[F(1,8)=5.88$, $p<.05$; Fig. 5, left]. Relative to saline infusions, NAc inactivation caused a small but significant decrease in the proportion of choices directed to the large/risky lever. Interestingly, under these conditions, NAc inactivation did not significantly affect response latencies $[F(1,8)=0.01$, n.s.; Table 2] or trial omissions $[F(1,8)=0.760$, n.s.; Table 2]. Locomotor activity was reduced following NAc inactivation, with this effect approaching statistical significance $[F(1,8)=4.56, p=.06$.; Table 2].

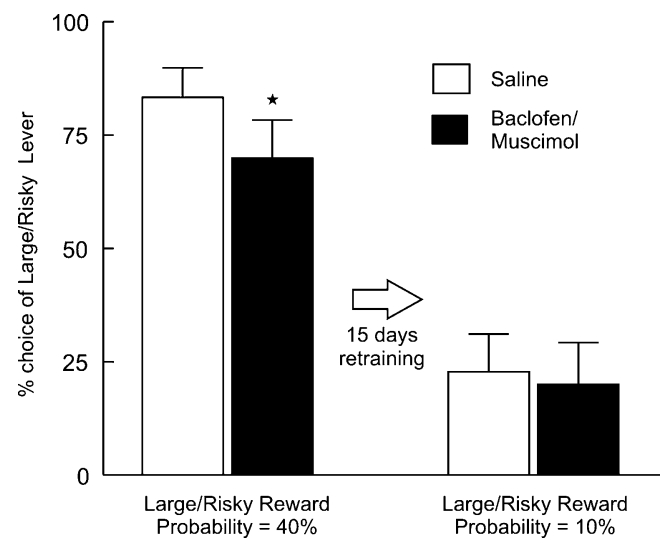

Fig. 5 Choice data for the fixed-probability risk task following inactivation of the NAc. Inactivation of the NAc reduced choice of the large/risky lever, but only when this option was more advantageous in the long term (four pellets with $40 \%$ probability, left). Rats were then trained for 15 days with the odds of obtaining the four-pellet reward reduced to $10 \%$. Under these conditions, inactivation of the NAc did not affect choice (right). The star denotes a significant main effect of treatment at $p<.05$
Table 2 Latency, locomotion, and trial omission data obtained from Experiment 2

\begin{tabular}{|c|c|c|c|c|}
\hline & \multicolumn{2}{|c|}{ Vehicle } & \multicolumn{2}{|c|}{ Inactivation } \\
\hline & $M$ & $S E$ & $M$ & $S E$ \\
\hline $40 \%$ : Response latency (s) & 0.74 & 0.1 & 0.82 & 0.2 \\
\hline Locomotion (beam breaks) & 1,205 & 131 & 851 & 96 \\
\hline Trial omissions & 0.11 & 0.1 & 1.00 & 0.9 \\
\hline 10\%: Response latency (s) & 0.58 & 0.1 & 0.57 & 0.1 \\
\hline Locomotion (beam breaks) & 1,078 & 150 & $889^{*}$ & 138 \\
\hline Trial omissions & 0.67 & 0.4 & 0.56 & 0.1 \\
\hline
\end{tabular}

${ }^{*} p<.05$ between vehicle and inactivation

For this experiment, we were unable to conduct an analysis of win-stay performance because this task had substantially fewer free-choice trials (20) than does the standard risk-discounting task (40). Because of this and the probabilistic nature of the reward delivery, there were considerably fewer instances where animals obtained the large/risky reward. Over the two test days, 3 rats obtained the large reward on 2 or fewer trials, and 1 rat did not obtain the large/risky reward at all (compare this to the standard risk-discounting task, where rats obtained the large reward on $12-15$ trials across tasks). This made calculation of win-stay ratios problematic, since their calculation requires at least 1 trial where subjects obtain the large/ risky reward, and at least 6-7 trials in order to be statistically meaningful and reliable. However, consistent with the findings of Experiment 1, lose-shift performance was unchanged by NAc inactivation $(M=.20 \pm .06)$ relative to saline infusions $(.19 \pm .06)[F(1,8)=0.02$, n.s.].

Rats were subsequently retrained on the task with the probability of reinforcement on the large/risky lever fixed to $10 \%$. Under these conditions, the small/certain option would yield more reward in the long term, and after 15 days of training, rats shifted their bias away from the large/risky lever, selecting it on $<30 \%$ of free-choice trials. They subsequently received a second round of counterbalanced microinfusions. Two rats receiving baclofen/muscimol for their first infusion required a second baseline training day for choice behavior to restabilize before their saline infusion. In contrast to the above-mentioned findings, under these conditions, inactivation of the NAc had no significant effect on choice $[F(1,8)=0.041$, n.s.; Fig. 5 , right]. For this phase of the experiment, rats chose the large/ risky lever on relatively few trials, and there were multiple instances where rats either did not choose this option at all or did not obtain the large/risky reward on any of the freechoice trials. This precluded us from calculating win-stay/ lose-shift ratios for this experiment. There were no significant effects of treatment on latencies $[F(1,8)=0.030, p>.05$; Table 2] or omissions $[F(1,8)=0.100, p>.05$; Table 2]. 
However, infusion of bacolfen/muscimol into the NAc again decreased locomotor activity relative to saline treatment $[F(1,8)=5.600, p<.05$; Table 2], indicating that the efficacy of these treatments to suppress neural activity was not diminished after repeated infusions.

In sum, these findings are consistent with those of the risk-discounting task, demonstrating that inactivation of the NAc biased choice away from the large/risky lever when that option had greater long-term value relative to the small/ certain option. However, when the odds of obtaining the larger reward were relatively low (i.e., the small/certain option had greater long-term value), inactivation of the NAc did not affect choice behavior.

Experiment 3: Effects of inactivation of the NAc or NAc shell on reward magnitude discrimination

In Experiment 1, inactivation of the NAc, or more selective inactivation of the shell, reduced preference for the larger, uncertain reward. In Experiment 3, a separate group of rats were trained on a simpler task, where they chose between two levers that delivered either one or four pellets, both with $100 \%$ probability.

NAc inactivations Eight rats were trained for 10 days on this task before receiving counterbalanced microinfusions of saline and baclofen/muscimol (125 ng each in $0.5 \mu \mathrm{l})$. Following saline infusions, rats displayed a very strong bias toward the larger reward, selecting this option on nearly $100 \%$ of the trials (Fig. 6a). Following infusions of baclofen/muscimol, rats continued to display a strong bias

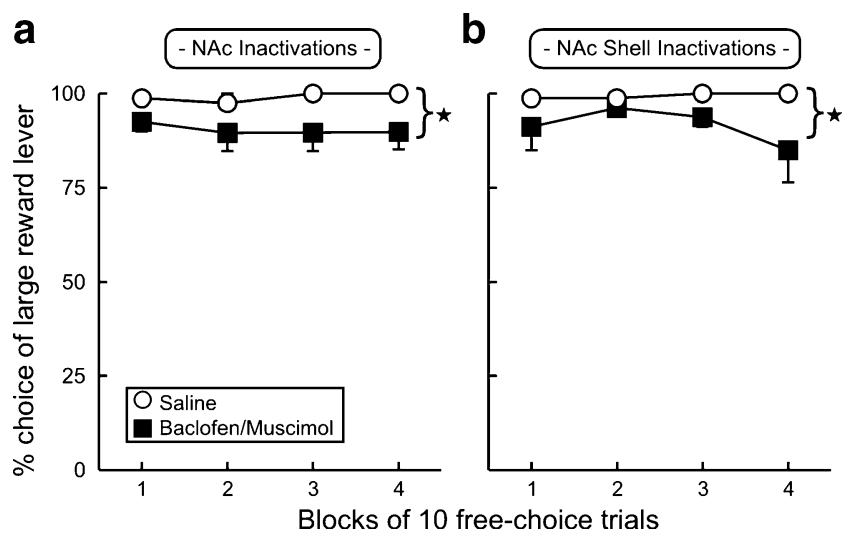

Fig. 6 Choice data for the reward magnitude discrimination task following (a)inactivation of the entire NAc and (b)more discrete inactivation of the NAc shell. Data are displayed as the proportions of choices of the large/risky lever in each of the four blocks of 10 free-choice trials. In both experiments, infusion of baclofen/muscimol induced a slight, but statistically significant, decrease in choices of the larger reward. Stars denote significant main effects of treatment at $p<.05$ toward the four-pellet option, yet the preference for this option was slightly reduced. Analysis of their choice behavior confirmed that the slight reduction in preference was statistically significant $[F(1,7)=6.34, p<.05]$. Trial omissions were unaffected by inactivation treatments $(M<1.0$ omissions for both treatments; $F(1,7)=2.05$, n.s.]. An equipment malfunction prevented acquisition of locomotor activity data from 4 rats on their saline infusion day. However, analysis of the locomotor data from the remaining 4 rats with complete data again revealed a significant decrease in activity induced by the inactivation treatment $(M=539 \pm 70)$ relative to saline $(M=938 \pm$ 103) $[F(1,3)=16.13, p<.05]$. Two rats displayed a prominent increase in response latencies after inactivation treatment, whereas the remaining 6 rats did not show this effect. As such, analysis of these data did not yield a statistically significant difference between treatment conditions $[F(1,7)=3.94$, n.s. $]$.

NAc shell inactivations A similar profile of choices was observed in another 8 rats that received inactivation of the NAc shell. Again, the rats selected the four-pellet option on almost all of the free-choice trials after saline infusions. However, infusions of baclofen/muscimol into the NAc shell $(75 \mathrm{ng}$ each in $0.3 \mu \mathrm{l})$ caused a slight, but statistically significant, decrease in choice of the larger reward $[F(1,7)=8.18, p<.05$; Fig. 6b]. There were no significant effects on latencies, locomotion, or omissions (all $F_{\mathrm{S}}<1.9$, n.s.). Thus, natural bias toward larger versus smaller rewards appears to be slightly blunted by inactivation of the NAc shell.

\section{Discussion}

The present data demonstrate that the NAc plays a critical role in biasing the direction of choice between smaller rewards and larger, probabilistic rewards and provide important new insight into the contribution of this nucleus to this form of decision making. Rather than inducing a general risk aversion or a disruption in reward valuation, inactivation of the NAc seems to reduce the bias toward larger-magnitude rewards that are either certain or uncertain when these options have greater long-term value. This effect appears attributable to a blunting of the impact that obtaining the larger reward has on biasing subsequent choices toward the risky option. Furthermore, our findings reveal that the NAc shell, rather than the core, seems to play a more critical role in guiding this form of decision making, whereas neural activity in the core exerts a greater influence over the latencies to make a choice. 
Contribution of the NAc to choice between rewards of different values

Using a probabilistic discounting task, we observed that the contribution of the NAc to choice between large/risky and small/certain options appeared to be greatest in the first three $(100 \%, 50 \%$, and $25 \%)$ trial blocks of this task, as has previously been observed following permanent lesions of the NAc using a similar discounting procedure (Cardinal \& Howes, 2005). During these trials, selection of the large/ risky option over blocks of 10 free-choice trials yielded either a greater or an equal reward relative to what could be obtained if the small/certain option was selected exclusively. As such, this option would have a greater long-term value. In contrast, during the $12.5 \%$ block, the low reinforcement probability outweighed the benefit of potentially receiving a larger reward, which would make the small/certain reward more advantageous. Although the analysis of the data did not produce a statistically significant Treatment $\mathrm{x}$ Trial Block interaction, a targeted analysis of these data confirmed that choice of the risky option was reduced during the first three blocks, but in the last, $12.5 \%$, block there was no apparent difference between the treatments in terms of choice. Note that during the latter block, rats still selected the large/risky lever on $50 \%-60 \%$ of trials after saline treatments, and NAc inactivation could in theory further bias choice away from this lever. Thus, it is unlikely that the lack of difference between treatment conditions during the last block reflects a floor effect. This being the case, even though the analysis revealed a significant overall decrease in choices of the large/ risky lever, this pattern of choice does not suggest that NAc inactivation uniformly increased risk aversion. One would expect that rational risk aversion would be associated with no change in choice in the early trial blocks, where there was little or no risk, and a decrease in choice of the large/risky lever in the blocks where choices were riskier. The different choice pattern observed here, with bias away from the large/risky option when it was advantageous but not when it was disadvantageous, suggests a different relationship between NAc functioning and choice behavior.

Further insight into the contribution of the NAc to risk discounting comes from a detailed analysis of choice behavior on trials following those in which animals chose risky and received the larger reward (win-stay) versus those where they selected the risky option and did not receive a reward (lose-shift). Under the control condition, animals chose the risky option on $>85 \%$ of trials following a win after selecting the large/risky option. Thus, obtaining the larger reward had a strong impact on determining how rats chose on the next trial. Conversely, on trials following a risky choice and loss, animals shifted to the small/certain option on $\sim 35 \%$ of subsequent trials. Inactivation of the
NAc or the shell subregion selectively reduced win-stay tendencies, demonstrating that obtaining a reward after a risky choice was less effective at biasing response selection toward the large/risky option on a subsequent trial. The lack of effect on lose-shift tendencies further argues against the notion that the effects of NAc inactivation on risky choice are due to increased risk aversion or a nonselective impairment in short-term memory for rewarded/nonrewarded events. Rather, these findings suggest that under conditions where reward delivery occurs with varying probabilities, the NAc plays a key role in using information about recently rewarded actions to bias the direction of subsequent ones. This notion is in keeping with data from neurophysiological recording studies of ventral striatal neurons in rats performing a probabilistic reversal task, where firing in a proportion of these cells conveyed information about both current and previous choices while the animal approached the reward location (Kim, Sul, Huh, Lee, \& Jung, 2009).

Experiment 2 further explored the effects of NAc inactivation on risky choice when the probabilistic option was either advantageous or disadvantageous. We used a simpler task, where the probability of obtaining the larger reward remained constant over a session. When the odds on the large/ risky lever were $40 \%$, rats displayed an appropriate bias toward this option. Under these conditions, NAc inactivation decreased choice of the large/risky lever in a manner consistent with the findings of Experiment 1. After being retrained on the task, where the odds of obtaining four pellets were now $10 \%$, rats displayed a preference for the small/ certain option. Importantly, under the latter conditions, inactivation of the NAc did not alter choice when the more valuable option was the smaller, certain reward. This is a key finding, as it shows that the neural activity in the NAc is not uniformly involved in biasing choice toward more valuable options in general. Were this the case, inactivation of the NAc would be expected to reduce choice of the small/certain option in favor of the large/risky one. Since this was not observed, it suggests that the contribution of this nucleus to decision making may be more selective, biasing choice toward options yielding larger yet uncertain rewards primarily when these options may provide more reward in the long term. In comparison, when options associated with smaller-magnitude rewards have a greater value in the long term, it appears that regions outside the ventral striatum may contribute to biasing choice toward these options.

The NAc and discrimination between larger and smaller rewards

In both of the above-mentioned experiments, inactivation of the NAc reduced choice of the option associated with the 
larger reward. To further clarify the nature of this effect, a separate group of animals were trained on a reward magnitude discrimination in order to determine whether inactivation of the NAc causes an irrational decrease in choice of a larger reward with no associated cost. Somewhat surprisingly, both inactivation of the NAc and more selective inactivation of the shell caused a subtle but statistically significant decrease in choice of the four-pellet option. Note that in this experiment, rats continued to display a strong bias toward the larger reward after inactivation of the NAc $(\sim 90 \%)$, but this was consistently lower than that displayed after saline infusions $(\sim 99 \%)$. Thus, it appears that the NAc shell can make a somewhat minor contribution to the normal bias animals have toward larger versus smaller rewards. It is unlikely that this effect is due to a somewhat minor deficit in spatial discrimination, because similar inactivation did not affect choice bias in Experiment 2 when the odds of obtaining the large reward were low. Interestingly, other researchers have failed to observe an effect of NAc lesions on magnitude discrimination using similar procedures (Cardinal \& Howes, 2005; Cardinal et al., 2000; Ghods-Sharifi \& Floresco, 2010). There may be a number of reasons for this discrepancy. First, most previous studies used permanent lesions and a between-subjects design, as opposed to the within-subjects design used in the present study. Given the relatively small difference between treatments we observed here, it is possible that these previous studies may have lacked sufficient statistical power to detect a significant effect (Cardinal \& Cheung, 2005; Cardinal \& Howes, 2005; Cardinal et al., 2000). Second, in previous lesion studies, animals were trained for a number of days postlesion, but only the data obtained in the latter part of retraining were analyzed, as compared with the present study, in which performance was examined on only the day of inactivation. Moreover, it is important to note that in one study (Cardinal \& Howes, 2005), NAc lesions did decrease choice of the larger reward, but this effect only trended toward statistical significance ( $p=.13$ in that study). Finally, some of these studies only investigated the contribution of the NAc core to this type of discrimination (Cardinal \& Howes, 2005; Ghods-Sharifi \& Floresco, 2010). The present results suggest that the shell may be the region of the ventral striatum that makes a more prominent contribution in biasing choice toward larger rewards.

Despite the finding that inactivation of the NAc or of its shell subregion slightly reduced bias toward larger rewards, it is important to emphasize that the effects on reward magnitude discrimination were considerably smaller than those observed on risk discounting. To elaborate on this point, note that the first block of the discounting task had the same reward contingencies as the magnitude discrimination task (four pellets vs. one, $100 \%$ probability for each).
Inactivation of the NAc prior to a risk-discounting session induced a $20 \%$ decrease in choice of the large reward option during this block (see Fig. 3a), as compared with a less than $10 \%$ reduction displayed by rats tested on reward magnitude discrimination (Fig. 6a). Although the reason for these differences is unclear, they are likely related to prior experiences that animals acquire during training on each task. Animals trained on the discounting task (but not the magnitude discrimination task) learn to expect that the likelihood of obtaining the larger reward will decrease over a session. Thus, the ability of NAc inactivation to markedly reduce choice of the large/risky option at the start of the discounting task may reflect an accelerated shift in bias away from this option in anticipation of changes in reward probability. In fact, inactivation of the NAc shell has been shown to accelerate behavioral shifts (Floresco et al., 2006). Thus, it appears that the NAc plays a small role in biasing choice between rewards of different magnitudes. However, the contribution of neural activity in this nucleus to choice behavior is amplified in situations requiring integration of multiple types of information regarding reward magnitude, changes in relative value, risk or other costs, and so forth, to bias the direction of behavior toward more favorable outcomes (Floresco et al., 2008).

Dissociable roles for the NAc shell and core in risk-based decision making

Infusion of a larger volume of a solution of GABA agonists $(0.5 \mu \mathrm{l})$ targeted at the central NAc altered multiple aspects of behavior on risk discounting (choice, response latencies, and locomotion). However, smaller infusion volumes $(0.3 \mu \mathrm{l})$ localized within the NAc shell or core each caused a more selective effect on either choice or response latencies/locomotion, respectively. This suggests that the more discrete infusions were effective at inducing relatively selective inactivation of either subregion. Furthermore, the fact that the larger infusion volume targeted at the border of shell/core induced changes in behavior that resembled the sum of the effects induced by inactivation of each subregion alone indicates that this procedure was effective at inactivating both subregions simultaneously.

Our finding that the shell rather than the core subregion of the NAc plays a more critical role in biasing choice was somewhat unexpected, in light of previous studies on the involvement of these subregions in other forms of cost/benefit decision making. Lesions to the NAc core, but not the shell, increased delay discounting, reducing the preference for larger, delayed rewards (Cardinal et al., 2001; Pothuizen et al., 2005). Similarly, lesions or inactivation of the NAc core, but not the shell, also reduced the tendency for animals to work harder (i.e., climb a scalable barrier, press a lever 
multiple times) to obtain a larger reward (Ghods-Sharifi \& Floresco, 2010; Hauber \& Sommer, 2009). When comparing these previous findings with the present data, it is important to keep in mind that with these other forms of decision making, even though selection of the large reward option incurs some form of cost (delay, effort), the subject is always guaranteed to obtain some reward after choice of either the high- or low-cost option. In comparison, during risk discounting, selection of the large/risky option may result in reward delivery or reward omission. Thus, the shell subregion of the ventral striatum may play a more prominent role in biasing response selection in situations offering greater uncertainty about obtaining reward. The seemingly selective role the NAc shell plays in these situations may be related to the proposed role of this subregion in the detection and reaction to novel environments or stimuli. Neural activity in the NAc shell has been proposed to be particularly sensitive to conditions involving cognitive processing related to novelty and/or uncertainty on tasks involving food neophobia or novel environments (Burns, Annett, Kelley, Everitt, \& Robbins, 1996; Rebec, Christensen, Guerra, \& Bardo, 1997; Wood \& Rebec, 2004). The probabilistic nature of reward delivery associated with the tasks used here shares similarities with novel situations, which are, by definition, associated with uncertainty about the rewarding or aversive consequences associated with future actions. The fact that NAc shell inactivations reduced the tendency to choose the large/risky option after receipt of a large reward on the preceding trial suggests that this subregion may play a key role in mitigating the impact that receipt of uncertain rewards has on subsequent choice.

Despite the fact that selective inactivation of the NAc core did not alter choice, we cannot rule out the possibility that this subregion also contributes to this form of decision making, given that larger inactivation of the NAc induced a numerically greater effect on risk discounting than did inactivation of the NAc shell alone. It may be that inactivation of the core by itself may not be sufficient to alter choice in these situations, but when combined with inactivation of the adjacent shell, the effect on behavior is amplified. Note, however, that infusion of GABA agonists into the core did significantly increase response latencies and reduce locomotor activity in a manner comparable to larger infusions across the core and shell. Previous work in our laboratory has shown similar effects on choice latencies following inactivation of the NAc core in rats performing an effort-discounting task (Ghods-Sharifi \& Floresco, 2010). This suggests that the neural activity within the NAc core facilitates the relative speed with which response selection occurs, ensuring that decisions are made in a timely fashion. This notion is supported by the neurophysiological observations of Roesch, Singh, Brown, Mullins, and Schoenbaum (2009). In that study, variations in NAc neural activity were correlated with the speed at which rats executed a response when they chose between rewards of different values. The present data confirm that these differences in activity do indeed impact the speed at which overt choices are made. Taken together, the present findings indicate that the NAc shell and core make dissociable contributions to risk-based decision making, with the shell making a more prominent contribution to the direction of choice and the core mediating the speed at which choices are made.

Neural mechanisms underlying NAc involvement in risk-based decision making

The present results complement those obtained from human functional imaging studies investigating NAc function with respect to risk-based decision making. For example, in a financial risk-taking study, Kuhnen and Knutson (2005) had subjects choose between a "safe" bond option that yielded smaller certain gains or a "risky" stock options that could potentially yield greater rewards or monetary losses, a task not dissimilar to the one used here. The authors reported that increased NAc activation preceded risky choices and risk-seeking mistakes. Our data suggest that this activity does indeed make a contribution to choice behavior under these conditions, biasing selection of a risky option associated with a larger reward. Other studies have implicated NAc activation in more general value discrimination, with changes in activity in this region being related to higher-valued rewards, based on indices of anticipated reward versus nonreward (Knutson, Fong, Adams, Varner, \& Hommer, 2001), anticipated gain magnitude (Knutson, Taylor, Kaufman, Peterson, \& Glover, 2005), and product preference (Knutson, Rick, Wimmer, Prelec, \& Lowenstein, 2007). Again, our inactivation data expand on these imaging study findings, suggesting that NAc activity may bias the direction of behavior when options associated with largermagnitude rewards may yield greater long-term gains.

Recordings from NAc neurons in awake, behaving animals provide further insight into the underlying cellular mechanisms through which activity in this nucleus may be related to decision making. Roesch et al., (2009) utilized a choice task in which relative cost (delay) or reward magnitude was manipulated. NAc neurons displayed greater increases in firing immediately prior to selection of higher-value options, indicating that these cells encode for the overall expected value of response outcomes. Phasic activity of mesoaccumbens dopamine also appears to encode aspects of reward value: Measurements of subsecond changes in mesoaccumbens dopamine via fast-scan cyclic voltametry have shown that the magnitude of rapid dopamine signaling is positively correlated with the relative value of an expected reward, when value was related to 
either reward magnitude (Gan, Walton, \& Phillips, 2010) or response cost (Day, Jones, Wightman, \& Carelli, 2010). These studies indicated that neuroelectrical and neurochemical activity in the NAc encodes an overall representation of value, including both costs and benefits, and ensures that these representations can influence overt behavior in a timely fashion. Admittedly, these studies did not explicitly investigate changes in NAc activity associated with choice between certain and uncertain rewards. Nevertheless, these neurophysiological studies, in combination with the present data, make it reasonable to propose that phasic changes in NAc activity prior to a choice may serve to bias the direction of behavior (via descending projections that feed into motor systems) toward options associated with larger, probabilistic rewards that have greater long-term value. Moreover, changes in NAc activity preceding a choice may in turn be modulated by the outcomes of previous choices (Kim et al., 2009).

When evaluating the relative contribution of the ventral striatum within the context of cortico-limbic-striatal circuitry that mediates decision making, it is important to emphasize that the NAc has been proposed to serve as a conduit through which cortical and limbic regions may bias the direction of behavior via its connections with motor systems (Floresco, Blaha, Yang, \& Phillips, 2001; Floresco, 2007; Mogenson et al., 1993). Thus, patterns of activity in upstream structures that process different aspects of information related to cost/benefit evaluations may drive NAc neural firing, via excitatory projections that can in turn influence the direction of choice. One brain region that may provide a critical source of input to the NAc is the basolateral amygdala. We have shown that inactivation of this nucleus also interferes with risk discounting, reducing choice of large/risky options (Ghods-Sharifi et al., 2009). Neural activity within the basolateral amygdala encodes information about differences in reward magnitudes, which in turn may represent part of a value signal that is integrated by the NAc (Belova, Paton, \& Salzman, 2008; Ernst et al., 2005; Pratt \& Mizumori, 1998; Smith et al., 2009). Moreover, both phasic activation of NAc neurons and approach behavior triggered by reward-related stimuli are blunted by basolateral amygdala inactivation, suggesting that this region provides a critical excitatory signal to the ventral striatum that influences the direction of ongoing behavior (Ambroggi, Ishikawa, Fields, \& Nicola, 2008; Jones et al., 2010). The medial prefrontal cortex is another input to the NAc that plays a critical role in risk discounting. Previous work in our laboratory suggested that this region of the frontal lobes monitors changes in reward probabilities to update value representations that facilitate efficient decision making (St. Onge \& Floresco, 2010). Thus, in the face of a choice, activity in these corticolimbic regions may convey different types of information (e.g., reward magnitude, changes in reward probability) that are integrated by ensembles of NAc neurons, which in turn may nudge the direction of behavior toward options that can provide more reward in the long term.

Acknowledgements This work was supported by a grant from the Canadian Institutes of Health Research (MOP 89861) to S.B.F. S.B.F. is a Michael Smith Foundation for Health Research Senior Scholar, and C.M.S. is the recipient of a University of British Columbia Graduate Fellowship.

\section{References}

Ambroggi, F., Ishikawa, A., Fields, H.L., \& Nicola, S.M. (2008). Basolateral amygdala neurons facilitate reward-seeking behavior by exciting nucleus accumbens neurons. Neuron, 59, 648-661. doi:10.1016/j.neuron.2008.07.004

Bari, A., Theobald, D.E., Caprioli, D., Mar, A.C., Aidoo-Micah, A., Dalley, J.W., \& Robbins, T.W. (2010). Serotonin modulates sensitivity to reward and negative feedback in a probabilistic reversal learning task in rats. Neuropsychopharmacology, 35, 1290-1301. doi:npp.2008.121/npp.2009.233

Belova, M.A., Paton, J.J., \& Salzman, C.D. (2008). Moment-to-moment tracking of state value in the amygdala. Journal of Neuroscience, 28, 10023-10030. doi:10.1523/JNEUROSCI.1400-08.2008

Brog, J. S., Salyapongse, A., Deutch, A. Y., \& Zahm, D. S. (1993). The patterns of afferent innervation of the core and shell in the "accumbens" part of the rat ventral striatum: Immunohistochemical detection of retrogradely transported fluoro-gold. The Journal of Comparative Neurology, 338, 255-278.

Burns, L. H., Annett, L., Kelley, A. E., Everitt, B. J., \& Robbins, T. W. (1996). Effects of lesions to the amygdala, ventral subiculum, medial prefrontal cortex, and nucleus accumbens on the reaction to novelty: Implication for limbic-striatal interactions. Behavioral Neuroscience, 110, 60-73.

Cardinal, R.N., \& Cheung, T.H. (2005). Nucleus accumbens core lesions retard instrumental learning and performance with delayed reinforcement in the rat. BMC Neuroscience, 6, 9. doi:10.1186/1471-2202-6-9

Cardinal, R.N., \& Howes, N.J. (2005). Effects of lesions of the nucleus accumbens core on choice between small certain rewards and large uncertain rewards in rats. BMC Neuroscience, 6, 37. doi:10.1186/1471-2202-6-37

Cardinal, R.N., Pennicott, D.R., Sugathapala, C.L., Robbins, T.W., \& Everitt, B.J. (2001). Impulsive choice induced in rats by lesions of the nucleus accumbens core. Science, 292, 2499-2501. doi:10.1126/science.1060818

Cardinal, R.N., Robbins, T.W., \& Everitt, B.J. (2000). The effects of damphetamine, chlordiazepoxide, alpha-flupenthixol and behavioural manipulations on choice of signalled and unsignalled delayed reinforcement in rats. Psychopharmacology, 152, 362-375. doi: $10.1007 / \mathrm{s} 002130000536$

Corbit, L.H., Muir, J.L., \& Balleine, B.W. (2001). The role of the nucleus accumbens in instrumental conditioning: Evidence of a functional dissociation between accumbens core and shell. Journal of Neuroscience, 21, 3251-3260. doi:0270-6474/01/213251-10

Day, J.J., Jones, J.L., Wightman, R.M., \& Carelli, R.M. (2010). Phasic nucleus accumbens dopamine release encodes effort- and delayrelated costs. Biological Psychiatry, 68, 306-309. doi:10.1016/j. biopsych.2010.03.026

Ernst, M., Nelson, E.E., Jazbec, S., McClure, E.B., Monk, C.S., Leibenluft, E., et al. (2005). Amygdala and nucleus accumbens in response to 
receipt and omission of gains in adults and adolescents. NeuroImage, 25, 1279-1291. doi:10.1016/j.neuroimage.2004.12.038

Floresco, S. B. (2007). Dopaminergic regulation of limbic-striatal interplay. Journal of Psychiatry \& Neuroscience, 32, 400-411.

Floresco, S.B., \& Whelan, J.M. (2009). Perturbations in different forms of cost/benefit decision making induced by repeated amphetamine exposure. Psychopharmacology, 205, 189-201. doi:10.1007/s00213-009-1529-0

Floresco, S.B., Blaha, C.D., Yang, C.R., \& Phillips, A.G. (2001). Modulation of hippocampal and amygdalar-evoked activity of nucleus accumben neurons by dopamine: Cellular mechanisms of input selection. Journal of Neuroscience, 21, 2851-2860. doi:0270-6474/01/212851-10

Floresco, S.B., Ghods-Sharifi, S., Vexelman, C., \& Magyar, O. (2006). Dissociable roles for the nucleus accumbens core and shell in regulating set shifting. Journal of Neuroscience, 26, 2449-2457. doi:10.1523/JNEUROSCI.4431-05.2006

Floresco, S.B., McLaughlin, R.J., \& Haluk, D.M. (2008). Opposing roles for the nucleus accumbens core and shell in cue-induced reinstatement of food-seeking behavior. Neuroscience, 154, 877 884.,doi:10.1016/j.neuroscience.2008.04.004

Floresco, S. B., Tse, M. T., \& Ghods-Sharifi, S. (2008). Dopaminergic and glutamatergic regulation of effort- and delay-based decision making. Neuropsychopharmacology, 33, 1966-1979.

Gan, J.O., Walton, M.E., \& Phillips, P.E.M. (2010). Dissociable cost and benefit encoding of future rewards by mesolimbic dopamine. Nature Neuroscience, 13, 25-27. doi:npp.2008.121/ nn. 2460

Ghods-Sharifi, S., \& Floresco, S. B. (2010). Differential effect on effort discounting induced by inactivations of the nucleus accumbens core or shell. Behavioral Neuroscience, 124, 179-191.

Ghods-Sharifi, S., St. Onge, J.R., \& Floresco, S.B. (2009). Fundamental contribution by the basolateral amygdala to different forms of decision making. Journal of Neuroscience, 29, 52515259. doi:10.1523/JNEUROSCI.0315-09.2009

Groenewegen, H. J., Berendse, H. W., Meredith, G. W., Haber, S. N., Voorn, P., Wolters, J. G., et al. (1991). Functional anatomy of the ventral, limbic-system innervated striatum. In P. Willner \& J. Scheel-Kruger (Eds.), The mesolimbic dopamine system: From motivation to action (pp. 19-59). New York: Wiley.

Hauber, W., \& Sommer, S. (2009). Prefrontostriatal circuitry regulates effort-related decision making. Cerebral Cortex, 19, 2240-2247. doi:10.1093/cercor/bhn241

Ikemoto, S., \& Panksepp, J. (1999). The role of nucleus accumbens dopamine in motivated behavior: A unifying interpretation with special reference to reward seeking. Brain Research Reviews, 31, 6-41. doi:10.1016/S0165-0173(99)00023-5

Jones, J.L., Day, J.J., Aragona, B.J., Wheeler, R.A., Wightman, R.M., \& Carelli, R.G. (2010). Basolateral amygdala modulates terminal dopamine release in the nucleus accumbens and conditioned responding. Biological Psychiatry, 67, 737-744. doi:10.1016/j. biopsych.2009.11.006

Kim, H., Sul, J.H., Huh, N., Lee, D., \& Jung, M.W. (2009). Role of striatum in updating values of chosen actions. Journal of Neuroscience, 29, 14701-14712. doi:10.1523/JNEUROSCI.272809.2009

Knutson, B., Fong, G. W., Adams, C. M., Varner, J. L., \& Hommer, D. (2001). Dissociation of reward anticipation and outcome with event-related fMRI. NeuroReport, 12, 3683-3687.

Knutson, B., Rick, S., Wimmer, G.E., Prelec, D., \& Lowenstein, G. (2007). Neural predictors of purchases. Neuron, 53, 147-156. doi:10.1016/j.neuron.2006.11.010

Knutson, B., Taylor, J., Kaufman, M., Peterson, R., \& Glover, G. (2005). Distributed neural representation of expected value. Journal of Neuroscience, 25, 4806-4812. doi:10.1523/JNEUR OSCI.0642-05.2005
Knutson, B., Wimmer, G.E., Kuhnen, C.M., \& Winkielman, P. (2008). Nucleus accumbens activation mediates the influence of reward cues on financial risk taking. NeuroReport, 19, 509-513. doi:10.1097/WNR.0b013e3282f85c01

Kuhnen, C.M., \& Knutson, B. (2005). The neural basis of financial risk taking. Neuron, 47, 763-770. doi:10.1016/j.neuron.2005.08.008

Marquis, J.P., Killcross, S., \& Haddon, J.E. (2007). Inactivation of the prelimbic, but not infralimbic, prefrontal cortex impairs the contextual control of response conflict in rats. European Journal of Neuroscience, 25, 559-566. doi:10.1111/j.1460-9568.2006.05295.x

Matthews, S. C., Simmons, A. N., Lane, S. D., \& Paulus, M. P. (2004). Selective activation of the nucleus accumbens during risk-taking decision making. NeuroReport, 15, 2123-2127.

Mobini, S., Body, S., Ho, M.Y., Bradshaw, C.M., Szabadi, E., Deakin, J. F, et al. (2002). Effects of lesions of the orbitofrontal cortex on sensitivity to delayed and probabilistic reinforcement. Psychopharmacology, 160, 290-298. doi:10.1007/s00213-001-0983-0

Mogenson, G. L., Brudzynski, S. M., Wu, M., Yang, C. R., \& Yim, C. Y. (1993). From motivation to action: A review of dopaminergic regulation of limbic $\rightarrow$ nucleus accumbens $\rightarrow$ ventral pallidum $\rightarrow$ pedunculopontine nucleus circuitries involved with limbic-motor integration. In P. W. Kalivas \& C. D. Barnes (Eds.), Limbicmotor circuits and neuropsychiatry (pp. 193-263). Boca Raton, FL: CRC Press.

Moreira, C.M., Masson, S., Carvalho, M.C., \& Brandão, M.L. (2007). Exploratory behaviour of rats in the elevated plus-maze is differentially sensitive to inactivation of the basolateral and central amygdaloid nuclei. Brain Research Bulletin, 71, 466-474. doi:10.1016/j.brainresbull.2006.10.004

Paxinos, G., \& Watson, C. (1998). The rat brain in stereotaxic coordinates (4th ed.). San Diego: Academic Press.

Pennartz, C. M., Groenewegen, H. J., \& Lopes Da Silva, F. H. (1994). The nucleus accumbens as a complex of functionally distinct neuronal ensembles: An integration of behavioral, electrophysiological, and anatomical data. Progressions in Neurobiology, 42, $719-761$.

Pothuizen, H.H., Jongen-Rêlo, A.L., Feldon, J., \& Yee, B.K. (2005). Double dissociation of the effects of selective nucleus accumbens core and shell lesions on impulsive-choice behaviour and salience learning in rats. European Journal of Neuroscience, 22, 2605-2616. doi:10.1111/j.1460-9568.2005.04388.x

Pratt, W. E., \& Mizumori, S. J. (1998). Characteristics of basolateral amygdala neuronal firing on a spatial memory task involving differential reward. Behavioral Neuroscience, 112, 554-570.

Rao, H., Korczykowski, M., Pluta, J., Hoang, A, \& Detre, J.A. (2008). Neural correlates of voluntary and involuntary risk taking in the human brain: An fMRI study of the Balloon Analog Risk Task (BART). NeuroImage, 42, 902-910. doi:10.1016/j.neuroimage.2008.05.046

Rebec, G.V., Christensen, J.R., Guerra, C., \& Bardo, M.T. (1997). Regional and temporal differences in real-time dopamine efflux in the nucleus accumbens during free-choice novelty. Brain Research, 776, 61-67. doi:10.1016/S0006-8993(97)01004-4

Roesch, M.R., Singh, T., Brown, P.L., Mullins, S.E., \& Schoenbaum, G. (2009). Ventral striatal neurons encode the value of the chosen action in rats deciding between differently delayed or sized rewards. Journal of Neuroscience, 29, 13365-13376. doi:10.1523/JNEUROSCI.2572-09.2009

Rudebeck, P.H., Walton, M.E., Smyth, A.N., Bannerman, D.M., \& Rushworth, M.F.S. (2006). Separate neural pathways process different decisions costs. Nature Neuroscience, 9, 1161-1168. doi:npp.2008.121/nn1756

Samanez-Larkin, G.R., Kuhnen, C.M., Yoo, D.J., \& Knutson, B. (2010). Variability in nucleus accumbens activity mediates agerelated suboptimal financial risk taking. Journal of Neuroscience, 30, 1426-1434. doi:10.1523/JNEUROSCI.4902-09.2010 
Smith, B.W., Mitchell, D.G., Hardin, M.G., Jazbec, S., Fridberg, D., Blair, R.J., \& Ernst, M. (2009). Neural substrates of reward magnitude, probability, and risk during a wheel of fortune decision-making task. NeuroImage, 44, 600-609. doi:10.1016/j. neuroimage.2008.08.016

St. Onge, J.R., Chiu, Y.C., \& Floresco, S.B. (2010). Differential effects of dopaminergic manipulations on risky choice. Psychopharmacology, 211, 209-221. doi:10.1007/s00213-010-1883-y

St. Onge, J.R., \& Floresco, S.B. (2009). Dopaminergic modulation of risk-based decision making. Neuropsychopharmacology, 34, 681-697. doi:npp.2008.121/npp. 2008.121

St. Onge, J.R., \& Floresco, S.B. (2010). Prefrontal cortical contribution to risk-based decision making. Cerebral Cortex, 20, 18161828. doi:10.1093/cercor/bhp250

van Duuren, E., van der Plasse, G., van der Blom, R., Joosten, R. N., Mulder, A. B., Pennartz, C. M., et al. (2007). Pharmacological manipulation of neuronal ensemble activity by reverse microdialysis in freely moving rats: A comparative study of the effects of tetrodotoxin, lidocaine, and muscimol. The Journal of Pharmacology and Experimental Therapeutics, $323,61-69$.
Walton, M.E., Kennerley, S.W., Bannerman, D.M., Phillips, P.E., \& Rushworth, M.F. (2006). Weighing up the benefits of work: Behavioral and neural analyses of effort-related decision making. Neural Networks, 19, 1302-1314. doi:10.1016/j.neunet.2006.03.005

Weiner, I. (2003). The "two-headed" latent inhibition model of schizophrenia: Modeling positive and negative symptoms and their treatment. Psychopharmacology, 169, 257-297. doi:10.1007/ s00213-002-1313-x

Winstanley, C.A., Theobald, D.E., Cardinal, R.N., \& Robbins, T.W. (2004). Contrasting roles of basolateral amygdala and orbitofrontal cortex in impulsive choice. Journal of Neuroscience, 24, 4718-4722. doi:10.1523/JNEUROSCI.5606-03.2004

Winstanley, C.A., Theobald, D.E., Dalley, J.W., \& Robbins, T.W. (2005). Interactions between serotonin and dopamine in the control of impulsive choice in rats: Therapeutic implications for impulse control disorders. Neuropsychopharmacology, 30, 669682. doi:10.1038/sj.npp.1300610

Wood, D.A., \& Rebec, G.V. (2004). Dissociation of core and shell singleunit activity in the nucleus accumbens in free-choice novelty. Behavioural Brain Research, 152, 59-66. doi:10.1016/j. bbr.2003.09.038 